\title{
Leptin and NUCB2/Nesfatin-1 in Acute Appendicitis
}

\author{
Unal Bakal1, Mehmet Saraç¹, Harun Ciftci², Tugay Tartar¹, Ahmet Kazez¹, \\ Suleyman Aydin ${ }^{3,4^{*}}$ \\ ${ }^{1}$ Department of Pediatric Surgery, School of Medicine, Firat University, Elazı̆̆, Turkey \\ ${ }^{2}$ Department of Medical Biochemistry, Ahievran University, Kirsehir, Turkey \\ ${ }^{3}$ Department of Medical Biochemistry (Firat Hormones Research Group), School of Medicine, Firat University, \\ Elazığ, Turkey \\ ${ }^{4}$ Illinois Institute of Technology, Chicago, IL, USA \\ Email: "saydin1@hotmail.com
}

Received 23 November 2015; accepted 22 December 2015; published 25 December 2015

Copyright () 2015 by authors and Scientific Research Publishing Inc.

This work is licensed under the Creative Commons Attribution International License (CC BY).

http://creativecommons.org/licenses/by/4.0/

(c) (i) Open Access

\section{Abstract}

Objective: Appetite loss is seen in $90 \%$ to $95 \%$ of patients with acute appendicitis; however, the cause of this symptom remains unknown. This study is performed to determine whether changes in the blood levels of two anorexigenic hormones, leptin and NUCB2/nesfatin-1, can help to diagnose acute appendicitis in children and whether these two parameters can distinguish acute appendicitis from abdominal pain. Methods: Sixty children with comparable ages and body mass indices are divided into three groups of 20 children each: those with acute appendicitis, those with abdominal pain, and controls. The blood sample with acute appendicitis is taken preoperatively (T1), and subsequent samples are taken 24 hrs postoperatively (T2) and 3 days postoperatively (T3). The blood sample with abdominal pain subjects is also taken in the corresponding times with those with acute appendicitis while blood sample from controls is only taken in the T1 corresponding time. Leptin and NUCB2/nesfatin-1 levels are measured by enzyme-linked immunosorbent assay. Results: The serum leptin levels are significantly higher preoperatively than postoperatively in all three groups. The NUCB2/nesfatin-1 levels at T1 in acute appendicitis are significantly higher than those at $\mathrm{T} 2$ in all three groups, but are restored at $\mathrm{T} 3$ to levels similar to those of controls. Neutrophil percentage has a sensitivity of $100 \%$, and specificity of $76.32 \%$, NUCB2/ nesfatin-1 level has a sensitivity of $47 \%$ and specificity of $95 \%$, and the leptin level has a sensitivity of $64 \%$ and specificity of $51 \%$ in the diagnosis of acute appendicitis. Conclusions: High preoperative leptin and NUCB2/nesfatin-1 levels may be a causative factor for appetite suppression observed in patients with acute appendicitis. High preoperative and low postoperative serum leptin and NUCB2/nesfatin-1 concentrations may serve as new candidate biomarkers that help to distinguish acute appendicitis from abdominal pain in children in addition to high CRP concentration, high WBC count, and neutrophilia.

"Corresponding author. 
Keywords

Leptin, NUCB2/Nesfatin-1, Acute Appendicitis, Biomarkers, Children

\section{Introduction}

Appendectomy to treat acute appendicitis is the most frequently performed emergent surgical procedure in all pediatric surgery departments. The clinical manifestations of appendicitis occur due to progression of the inflammatory process, which begins with obstruction of the lumen of the vermiform appendix. The diagnosis of acute appendicitis is currently based on clinical findings. The first sign of acute appendicitis is usually pain that begins in the mid-abdomen and sharpens as it moves toward the right lower abdomen. Nausea and/or vomiting develop soon after the abdominal pain begins, and subsequent abdominal swelling, inability to pass gas, high body temperature $\left(>38^{\circ} \mathrm{C}\right)$, and loss of appetite follow. Severe cramps, painful urination, and constipation or diarrhea with gas may also be seen in patients with acute appendicitis. These clinical symptoms result in confusion between acute appendicitis and a number of other conditions, especially nonspecific abdominal pain [1]. Various biochemical markers such as the C-reactive protein (CRP) level, neutrophil percentage, white blood cell (WBC) count, serum 5-hydroxyindoleacetic acid and neutrophil gelatinase-associated lipocalin levels, and various scoring systems are currently used to aid the clinical diagnosis [2]-[6]. Diagnosis of appendicitis during its acute period is important to easily treat this disease and to prevent complications. Despite advances and developments in medicine, we still encounter problems such as perforation, negative appendectomy, mortality, morbidity, and long hospital stays in patients with acute appendicitis [7].

The serum levels of ghrelin (an orexigenic hormone) [8] and procalcitonin (an anorexigenic hormone) [9] are recently studied in patients with acute appendicitis to investigate the loss of appetite associated with this disease. These studies reveal that acute appendicitis is associated with both hormones. However, a number of orexigenic and anorexigenic molecules other than ghrelin and procalcitonin are synthesized in the body. Leptin, for example, is an anorexigenic molecule that is synthesized in adipocytes as well as many other peripheral tissues. An increased leptin level causes loss of appetite and decreased food intake and controls energy consumption [10] [11]. Leptin is also involved in the regulation of food intake and body weight by promoting fat oxidation over carbohydrate oxidation. In fact, leptin has been confirmed to be a novel indicator of the fat content. Moreover, the leptin concentration is reportedly associated with obesity, obesity-associated insulin resistance, obesity-related nonalcoholic fatty liver disease, and diabetes and serves as a biomarker for many other metabolic diseases [12].

However, its relationship with the loss of appetite seen in patients with acute appendicitis remains unclear.

The 82-amino acid protein from nucleobindin-2 gene (NUCB2) is nesfatin-1 (another anorexigenic hormone) [13] [14]. The relationship between this hormone and acute appendicitis is also unclear. NUCB2 contains the epitope that the antibody is raised against; it is very likely that it is also reacted by the nesfatin- 1 immunoreactivity. For this reason, currently available nesfatin-1 enzyme-linked immunosorbent assay (ELISA) kits detect both NUCB2 and nesfatin-1 concentrations in biological samples. Therefore, nesfatin-1 is termedNUCB2/nesfatin-1 throughout the present report [13]. Intracerebroventricular injection of nesfatin-1 in rats is shown to inhibit the appetite, which leads to subsequent weight loss, while the antibody-neutralizing nesfatin- 1 treatment resulted in increased food intake (14)NUCB2/nesfatin-1 levels is associated with obesity, obesity-associated insulin resistance, obesity-related nonalcoholic fatty liver disease, thermogenesis, diabetes, aging, pediatric obstructive sleep apnea, and many other metabolic diseases [13], and the intensity of exercise [15]. High NUCB2/nesfatin-1 levels are found in the satiety center as well as in the paraventricular, supraoptic, arcuate, and lateral nuclei of the hypothalamus [14]. Studies have also shown that NUCB2/nesfatin-1 is responsible for regulation of appetite and metabolic processes in the hypothalamus [13]-[15]. In fact, NUCB2/nesfatin-1 has been confirmed to be a potential blood-based protein biomarker for several diseases, particularly epilepsy [16].

Thus, it has been hypothesized that nesfatin-1 may play a role in the loss of appetite of unknown origin seen in patients with acute appendicitis. With this background, the present investigation was designed to assess whether serum leptin and NUCB2/nesfatin-1 levels play a role in the diagnosis of acute appendicitis, can distinguish acute appendicitis from abdominal pain, and help in the diagnosis of acute appendicitis. 


\section{Materials and Methods}

The ethics committee of Firat University Faculty of Medicine approved this study (2 May 2013, decree no. 05). All enrolled 60 children in this study were admitted through the department of Pediatric Surgery of Firat University and they were divided into 3 groups, aged $7-12$ years with a similar body mass index: those with acute appendicitis (Group 1, $n=20$ ), those with abdominal pain (Group 2, $n=20$ ), and healthy controls (Group 3, $n=$ 20). Diagnoses were confirmed by using either computed tomography (CT) without contrast medium or ultrasound after physical exam together with WBC count. Then, only acute appendicitis (Group 1) underwent surgery. The control group was composed of healthy children who were suspected for cryptorchidism, inguinal hernia, phimosis and vulvar fusion (but they were diagnosed as a negative) at the pediatric surgery department. Also, the age and cognitive skills of the children prevented them from using a visual analog scale to evaluate their appetite; their parents stated whether the children had a normal appetite or loss of appetite. The parents of all children in this study provided written informed consent for participation. Blood samples in Group 1 were obtained preoperatively (T1), 24 h postoperatively (T2), and 3 days postoperatively (T3). Patients with acute appendicitis are usually admitted to our hospital in the afternoon; thus, blood samples in Groups 2 were obtained during the same time period (at about 3:00 pm) to avoid any differences related to the circadian rhythm. However, blood samples in Groups 3 (Control) were taken once (T1), but the T1 hormonal alteration levels were alsoused for T 2, and T3) to compare differences easily with and without abdominal pain or acute appendicitis.

The WBC count, neutrophil percentage, CRP level, erythrocyte sedimentation rate (ESR), and body mass index of all patients were determined upon hospital admission. The blood samples were placed into tubes containing 500 Kallikrein units (KIU) of aprotinin. The taken blood was centrifuged within 30 min. at 4000 rpm for 5 min. Then, the samples were stored at $-80^{\circ} \mathrm{C}$ until analysis of leptin and NUCB2/nesfatin- 1 levels.

\subsection{Analytic Measurements}

Serum leptin levels were determined by ELISA using a DIA source Leptin-EASIA Kit (DIA source Immuno Assays S.A., Louvain-la-Neuve, Belgium) according to the manufacturer's instructions. The minimum detection limit of leptin was $0.04 \mathrm{ng} / \mathrm{ml}$, the intra-assay variation was $1.5 \pm 0.2 \mathrm{ng} / \mathrm{ml}$, and the interassay variation was $5.9 \pm$ $0.6 \mathrm{ng} / \mathrm{ml}$. NUCB2/nesfatin-1 (cat no. E90242Hu) was measured with an ELISA kit for NUCB2/nesfatin-1 (NES1) (USCN Life Science, Inc., Wuhan, China) according to the manufacturer's instructions. The minimum detection limit for NUCB2/nesfatin-1 was $272.5 \mathrm{pg} / \mathrm{ml}$, the intra-assay variation was $<10 \%$, and the interassay variation was $<12 \%$. All samples were studied at $450 \mathrm{~nm}$ using a Chro Mate 4300 ELISA Reader (Awareness Technology, Inc.). The WBC count and neutrophil percentage were measured with an ABX Pentra DX SPS Evolution device (Horiba, Ltd., Kyoto, Japan), the ESR was measured with a VacuplusESR-120 Full Automated ESR analyzer, and the CRP level was measured with a Siemens Dade Behring BN II Nephelometer device.

\subsection{Statistical Analysis}

The data were analyzed with IBM SPSS Statistics for Windows, version 21 (IBM Corp., Armonk, NY). Tukey's honestly significant difference (HSD) test was employed for intergroup comparisons of the NUCB2/nesfatin-1 and leptin levels, CRP level, WBC count, neutrophil percentage, and ESR at admission. NUCB2/nesfatin-1 and leptin levels measured preoperatively, 24 hrs postoperatively, and 72 hrs postoperatively were also compared using Tukey's HSD test. A $p$ value of $<0.05$ was considered statistically significant. The specificity and sensitivity of leptin and NUCB2/nesfatin-1 measurements were determined using receiver operating characteristic (ROC) curves.

\section{Results}

No statistically significant differences were observed in age (7 - 12 years) or body mass index among Groups 1 , 2, and 3 (BMI: 15.5 - 24.9), and sex among Groups 1 (8 Females/12 Males), 2 (9 Females/11 Males), and 3 (10 Females/10 Males). The biochemical parameters of the patients are presented in Table 1 . The CRP level, WBC count, neutrophil percentage, and ESR were significantly higher in Groups 1 and 2 than in Group 3 (Table 1).

The leptin levels in Group 1 at T1 were higher than those in Groups 2 and 3. The leptin levels upon hospital admission (T1) in Groups 1, 2, and 3 were $2.290 \pm 0.800,1.304 \pm 0.200$, and $0.643 \pm 0.100 \mathrm{ng} / \mathrm{ml}$, respectively (Figure 1). When a cut-off leptin level of $0.271 \mathrm{ng} / \mathrm{ml}$ was chosen, its sensitivity and specificity for diagnosis of acute appendicitis were 64\% and 51\%, respectively, at a 95\% confidence interval (Figure 2). 
Table 1. Changes in biochemical parameters in patients with acute appendicitis, patients with abdominal pain, and healthy controls.

\begin{tabular}{cccc}
\hline Parameters & Acute appendicitis & Abdominal pain & Control \\
\hline CRP level $(\mathrm{mg} / \mathrm{dl})$ & $5.17^{\mathrm{a}}$ & 1.23 & 0.80 \\
WBC count $(\mathrm{K} / \mathrm{\mu l})$ & $16.72^{\mathrm{b}}$ & 11.65 & 7.74 \\
Neutrophil $(\%)$ & $85.56^{\mathrm{c}}$ & 61.85 & 54.76 \\
ESR $(\mathrm{mm} / \mathrm{h})$ & $26.00^{\mathrm{d}}$ & 14.40 & 10.15 \\
\hline
\end{tabular}

Acute appendicitis vs. abdominal pain vs. controls $\left({ }^{\mathrm{a}, \mathrm{b}, \mathrm{c}, \mathrm{d}} p<0.05\right)$. CRP, C-reactive protein; WBC, white blood cell; ESR, erythrocyte sedimentation rate.

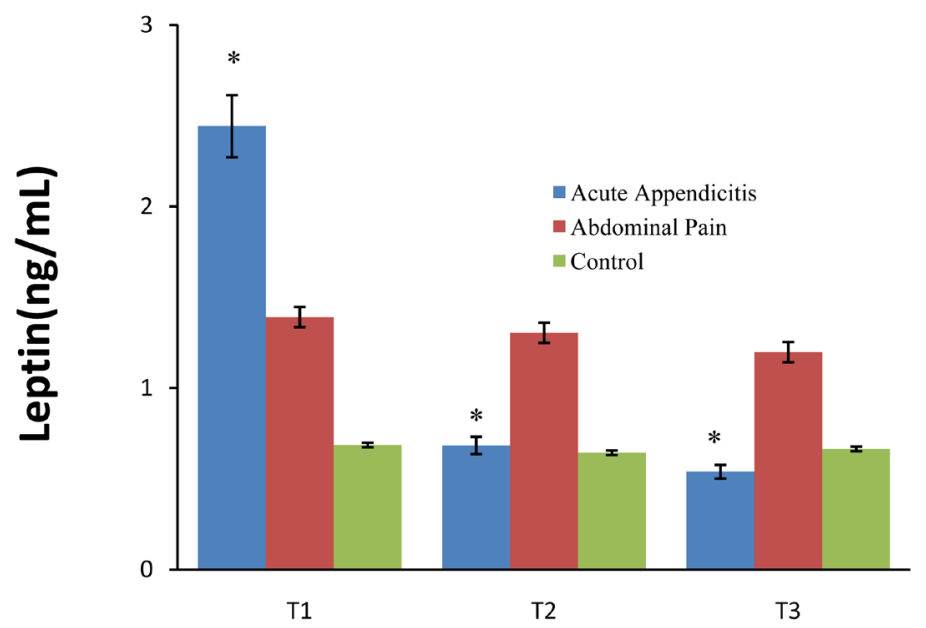

Figure 1. Preoperative (T1), 24-h postoperative (T2), and 72-h postoperative (T3), leptin levels in children with acute appendicitis (Group 1), children with abdominal pain (Group 2), and healthy controls (Group 3, the same concentration of leptin analysis at T1 was also used for T2 and T3). Acute appendicitis vs. abdominal pain vs. controls $\left({ }^{*} p<0.05\right)$.

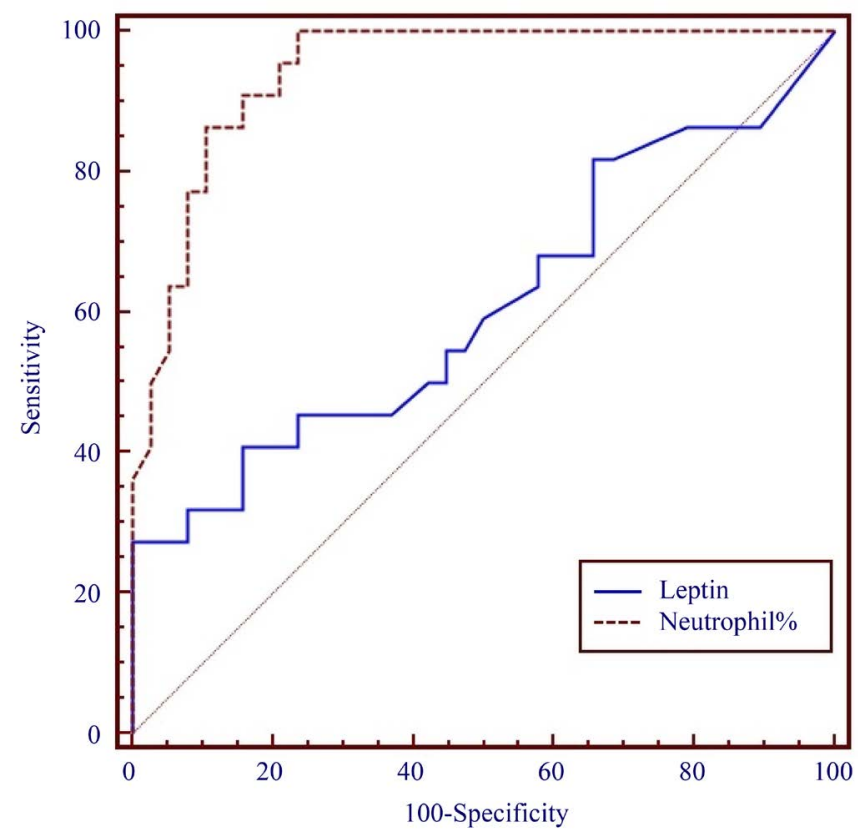

Figure 2. Sensitivity and specificity of preoperative serum leptin levels and neutrophil\% for diagnosis of acute appendicitis in children. 
The NUCB2/nesfatin-1 level at T1 was higher in Group 1 than in Groups 2 and 3. The NUCB2/nesfatin-1 levels in Groups 1, 2, and 3 at T1 were $11.852 \pm 7.700,7.344 \pm 2.100$, and $8.976 \pm 1.200 \mathrm{ng} / \mathrm{ml}$, respectively. However, the NUCB2/nesfatin-1 level in Group 1 was significantly higher at T2 than T1. The level at T3 was similar to that in Group 3 (Figure 3). When the cut-off value $>65.4$ neutrophil percentage was chosen, its sensitivity and specificity were $100 \%$ and $76.32 \%$ respectively, at a 95\% confidence interval (Figure 2 and Figure 4). Whena NUCB2/nesfatin-1 cut-off level of $8.882 \mathrm{ng} / \mathrm{ml}$ was chosen, its sensitivity and specificity were $47 \%$ and 95\%, respectively, at a 95\% confidence interval (Figure 4). The sensitivity and specificity of NUCB2/nesfatin-1 were higher than those of leptin for the diagnosis of acute appendicitis in children (Figure 2 and Figure 4). In the acute appendicitis positive correlation was found between leptin and neutrophil $(r=0.407, p=0.05)$. Similar correlations were also found between NUCB2/Nesfatin-1 and neutrophil $(r=0.401, p=0.05)$.

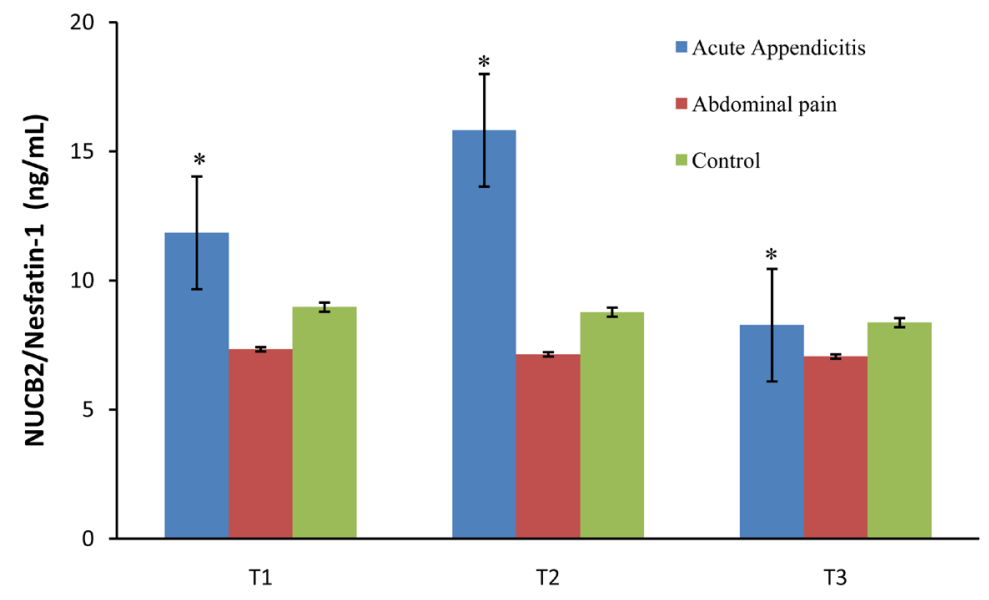

Figure 3. Preoperative (T1), 24-h postoperative (T2), and 72-h postoperative (T3), NUCB2/nesfatin-1 levels in children with acute appendicitis (Group 1), children with abdominal pain (Group 2), and healthy controls (Group 3; the same concentration of NUCB2/nesfatin-1 analysis at timing (T1) was also used for T2 and T3). Acute appendicitis vs. abdominal pain vs. controls $\left({ }^{*} p<0.05\right)$.

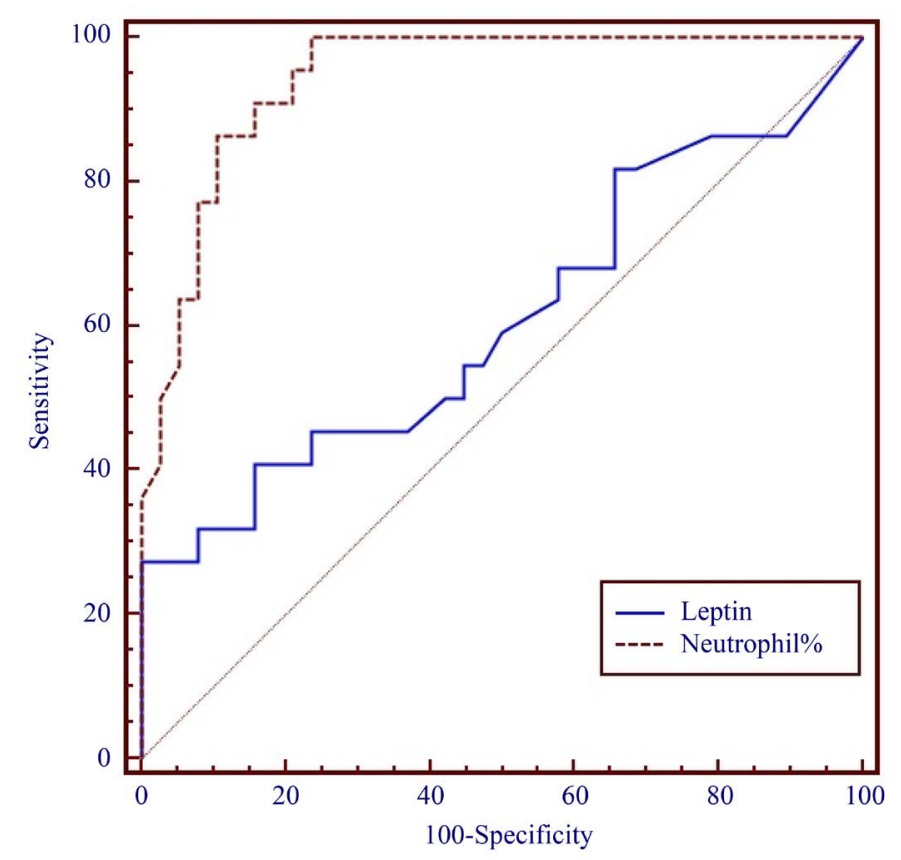

Figure 4. Sensitivity and specificity of preoperative serum NUCB2/nesfatin-1 levels and neutrophil \% for diagnosis of acute appendicitis in children. 


\section{Discussion}

Appendicitis is characterized by inflammation of the appendix vermiformis, which is a $<8$-mm-wide and 8- to 10 -cm-long tube of tissue that extends from the cecum in children. Acute appendicitis is the most frequent disease worldwide that requires emergent surgical intervention. Failure of doctors to recognize the symptoms of acute appendicitis sometimes results in death of children with this condition. Its clinical findings are usually confused with those of other diseases, especially those characterized by nonspecific abdominal pain. The diagnosis is usually based on the patient's history and physical examination findings. A rapid and correct diagnosis is expected to decrease the morbidity rate. However, no specific and sensitive biochemical parameters are available for the diagnosis of acute appendicitis. The combination of a high CRP concentration, high WBC count, D-lactate levels and neutrophilia is currently used to diagnose appendicitis and exhibits 95\% accuracy [7] [17]-[21]. However, the present study indicates that although the neutrophil count, CRP concentration, and WBC count were high in patients with acute appendicitis, these parameters were not sensitive and specific enough to distinguish acute appendicitis from abdominal pain (Table 1).

Ghrelin is an orexigenic hormone, and loss of appetite is a well-known symptom of acute appendicitis. Therefore, the sensitivity and specificity of the serum ghrelin level for detection of acute appendicitis have recently been evaluated. Cetinkaya et al. [8] found that the preoperative ghrelin levels in saliva and serum were significantly lower than the postoperative levels in both patients undergoing appendectomy and control patients. They concluded that low serum ghrelin levels in patients with acute appendicitis might aid in the diagnosis of this condition. More recently, the acylated and des-acylated ghrelin levels were investigated in patients with mild pancreatitis and those with severe pancreatitis. The researchers found no statistically significant differences in the acylated or des-acylated ghrelin levels between the two groups either at presentation or following the initiation of oral feeding [15]. In the present study, however, we hypothesized that anorexigenic hormones are more important than orexigenic hormones in the etiopathogenesis of acute appendicitis. High preoperative leptin and NUCB2/nesfatin-1 concentrations in patients with appendicitis might be a causative factor for the appetite suppression observed in patients with acute appendicitis. Therefore, we investigated whether in addition to the neutrophil count, CRP level, and WBC count, biomarkers of appetite suppression such as leptin and NUCB1/nesfatin-1 should be measured in patients who present with suspicion of acute appendicitis based on clinical findings.

To the best of our knowledge, this study is the first to show that the levels of the anorexigenic hormones leptin and NUCB2/nesfatin-1 are higher in preoperative serum samples of patients with acute appendicitis than in the same samples of patients with abdominal pain and of healthy controls. These two hormones might be rapidly released into the bloodstream from their site of synthesis, allowing them to be detected in the blood early after the onset of acute appendicitis and thus helping to distinguish acute appendicitis from abdominal pain. We thus assumed that the appetite loss in patients with acute appendicitis was caused by the higher leptin and NUCB2/ nesfatin-1 levels in these patients. In a previous study, intracerebroventricular injection of nesfatin-1 inhibited appetite and caused subsequent weight loss, while antibody-neutralizing nesfatin-1 treatment resulted in increased food intake [14]. Leptin is mainly synthesized in adipose tissues and also is synthesized in the hypothalamus and decreases food consumption by decreasing Agouti-related protein and neuropeptide Y levels [10]. Other studies have also shown that leptin and NUCB2/nesfatin-1 are responsible for appetite and metabolic regulation in the hypothalamus [14] [22]. Therefore, in the present study, we assumed that a NUCB2/nesfatin-1 level above the physiological concentrations may be associated with the loss of appetite seen in children with acute appendicitis. It is also known that appendicitis may be caused by gram-negative, LPS-containing bacteria. LPS in turn has been shown to increase Nucb2/nesfatin-1 plasma levels in rats, most likely due to release from gastric endocrine cells [23].

Another finding of the present study was the significantly higher 24-h postoperative than preoperative serum NUCB2/nesfatin-1 levels in patients with acute appendicitis. We consider that this increase in the NUCB2/nesfatin-1 levels was caused by additional surgical stress. Similarly, Cetinkaya et al. [8] reported an increase in ghrelin levels after surgical stress had subsided. It has been also shown that abdominal surgery in rats induces a potent decrease of ghrelin levels [24]. Since an opposite regulation of ghrelin and nesfatin-1 has been known. If so, this could account for the post-surgical increase in nesfatin levels. Over all, these findings indicate that surgical stress might affect the endocrine system.

Comparison of the sensitivity and specificity should be performed at a point on the individual ROC curves where the likelihood ratios are equivalent and clinically meaningful. Therefore, in this work, we also used ROC 
curves to analyze the sensitivity and specificity of the leptin and NUCB2/nesfatin-1 levels in patients with acute appendicitis. It was found that neutrophil percentage had a sensitivity of $100 \%$ and specificity of $76.32 \%$ at a confidence interval of $95 \%$ when the cut-off value was set at $>65.4$ neutrophil percentage. We found that NUCB2/nesfatin-1, rather than leptin, seems to have diagnostic significance for acute appendicitis because it exhibited $47 \%$ sensitivity and $95 \%$ specificity at a confidence interval of $95 \%$, when the cut-off value was set at $8.882 \mathrm{ng} / \mathrm{ml}$. However, when the cut-off value was set at $0.271 \mathrm{ng} / \mathrm{ml}$, leptin had a sensitivity of $64 \%$ and specificity of $51 \%$ at a confidence interval of $95 \%$. The diagnostic performance of each acute appendicitis markers, especially nesfatin-1 was superior to neutrophil percentage (76.32\%) owing to its (nesfatin-1) high specificity (95\%) properties.

\section{Study Limitations}

We must interpret the present findings cautiously because of the following limitations. The main limitation of our study is the small number of patients and no power calculation has been done. Another limitation is that this is a single-center study. The results should be confirmed in a prospective multicenter study involving larger numbers of patients.

However, these limitations do not diminish the value of this study. The patients with acute appendicitis are presented with significantly higher serum leptin and NUCB2/nesfatin-1 levels than patients with abdominal pain and control patients. Leptin and especially NUCB2/nesfatin-1 clearly have higher diagnostic accuracy if it is used in patients with acute appendicitis presenting to the emergency and pediatric surgery departments. Additionally, these newer tests provide a major addition to measurement of the CRP level and WBC count. Furthermore, before surgical treatment of acute appendicitis, measurement of the serum leptin and NUCB2/nesfatin-1 levels may help surgeons to distinguish acute appendicitis from nonspecific abdominal pain. No extra logistics are necessary for the performance of these tests, and their cost is similar to that of the currently used CRP and WBC tests. However, measurement of leptin and NUCB2/nesfatin-1 in combination with measurement of CRP and WBC is highly accurate for the diagnosis of acute appendicitis in children. Therefore, in addition to high CRP concentration, high WBC count, D-lactate levels and neutrophilia and also a combination of the leptin and NUCB2/nesfatin-1 concentrations can be used as a screening test for the diagnosis of acute appendicitis based on the present results, which show promise distinguishing acute appendicitis from abdominal pain. However, drawing conclusions from the results of the confirmation are important on a larger scale with different medical center before using those parameters for diagnostic purpose of distinguishing acute appendicitis from abdominal pain.

\section{Acknowledgements}

The authors express their gratitude to Emrah H. Firat for his contributions to the statistical analysis and to Habibe Kazez for helping us to draw the figures.

\section{Conflict of Interest}

The authors declare that they have no conflict of interest.

\section{Author Contributions}

UB and MS clinical data collection, patients' treatment, follow-up, and helped to draft the manuscript, HC and TT participated in the design of the study and carried out the graphic design of figures, AK and SA wrote and reviewed the manuscript, and they were the guarantor of the integrity of the study. All authors participated in the definition of intellectual content. All authors read and approved the final manuscript.

\section{References}

[1] Nance, M.L., Adamson, W.T. and Hedrick, H.L. (2000) Appendicitis in the Young Child: A Continuing Diagnostic Challenge. Pediatric Emergency Care, 16, 160-162. http://dx.doi.org/10.1097/00006565-200006000-00005

[2] Alvarado, A. (1986) A Practical Score for the Early Diagnosis of Acute Appendicitis. Annals of Emergency Medicine, 15, 557-564. http://dx.doi.org/10.1016/S0196-0644(86)80993-3 
[3] Apak, S., Kazez, A., Ozel, S.K., Ustundag, B., Akpolat, N. and Kizirgil, A. (2005) Spot Urine 5-Hydroxyindoleacetic Acid Levels in the Early Diagnosis of Acute Appendicitis. Journal of Pediatric Surgery, 40, 1436-1439. http://dx.doi.org/10.1016/j.jpedsurg.2005.05.042

[4] Bakal, U., Sarac, M., Ciftci, H., Tartar, T. and Kazez, A. (2014) Neutrophil Gelatinase-Associated Lipocalin (NGAL) May Be Support in the Diagnosis of Acute Appendicitis. 32nd National Congress of Pediatric Surgery in Trabzon, Turkey, 17-20 September 2012, 138.

[5] Branescu, C., Serban, C., Savlovschi, C., Dascalu, A.M. and Kraft, A. (2012) Lipopolysaccharide Binding Protein (L.B.P.)-An Inflammatory Marker of Prognosis in the Acute Appendicitis. Journal of Medicine and Life, 5, 342-347.

[6] Rodriguez-Sanjuan, J.C., Martin-Parra, J.I., Seco, I., Garcia-Castrillo, L. and Naranjo, A. (1999) C-Reactive Protein and Leukocyte in the Diagnosis of Acute Appendicitis in Children. Diseases of the Colon \& Rectum, 42, 1325-1329. http://dx.doi.org/10.1007/BF02234223

[7] Flum, D.R. and Koepsell, T. (2002) The Clinical and Economic Correlates of Misdiagnosed Appendicitis: Nationwide Analysis. Archives of Surgery, 137, 799-804. http://dx.doi.org/10.1001/archsurg.137.7.799

[8] Cetinkaya, Z., Aydin, S., Cerrahoglu, Y.Z., Ayten, R., Erman, F. and Aygen, E. (2009) Changes in Appetite Hormone (Ghrelin) Levels of Saliva and Serum in Acute Appendicitis Cases before and after Operation. Appetite, 52, 104-107. http://dx.doi.org/10.1016/j.appet.2008.09.001

[9] Sand, M., Trullen, X.V., Bechara, F.G., Pala, X.F., Sand, D., Landgrafe, G. and Mann, B. (2009) A Prospective Bicenter Study Investigating the Diagnostic Value of Procalcitonin in Patients with Acute Appendicitis. European Surgical Research, 43, 291-297. http://dx.doi.org/10.1159/000232939

[10] Ahima, R.S. and Flier, J.S. (2000) Leptin. Annual Review of Physiology, 62, 413-437. http://dx.doi.org/10.1146/annurev.physiol.62.1.413

[11] Mc Conway, M.G., Johnson, D., Kelly, A., Griffin, D., Smith, J. and Wallace, A.M. (2000) Differences in Circulating Concentrations of Total, Free and Bound Leptin Relate to Gender and Body Composition in Adult Humans. Annals of Clinical Biochemistry, 37, 717-723. http://dx.doi.org/10.1258/0004563001899771

[12] Farr, O.M., Gavrieli, A. and Mantzoros, C.S. (2015) Leptin Applications in 2015: What Have We Learned about Leptin and Obesity? Current Opinion in Endocrinology, Diabetes and Obesity, 22, 353-359. http://dx.doi.org/10.1097/MED.0000000000000184

[13] Aydin, S. (2013) Multi-Functional Peptide Hormone NUCB2/Nesfatin-1. Endocrine, 44, 312-325. http://dx.doi.org/10.1007/s12020-013-9923-0

[14] Oh, I., Shimizu, H., Satoh, T., Okada, S., Adachi, S., Inoue, K., Eguchi, H., Yamamoto, M., Imaki, T., Hashimoto, K., Tsuchiya, T., Monden, T., Horiguchi, K., Yamada, M. and Mori, M. (2006) Identification of Nesfatin-1 as a Satiety Molecule in the Hypothalamus. Nature, 443, 709-712. http://dx.doi.org/10.1038/nature05162

[15] Mohebbi, H., Nourshahi, M., Ghasemikaram, M. and Safarimosavi, S. (2015) Effects of Exercise at Individual Anaerobic Threshold and Maximal Fat Oxidation Intensities on Plasma Levels of Nesfatin-1 and Metabolic Health Biomarkers. Journal of Physiology and Biochemistry, 71, 79-88. http://dx.doi.org/10.1007/s13105-015-0383-2

[16] Aydin, S., Dag, E., Ozkan, Y., Arslan, O., Koc, G., Bek, S., Kirbas, S., Kasikci, T., Abasli, D., Gokcil, Z., Odabasi, Z. and Catak, Z. (2011) Time-Dependent Changes in the Serum Levels of Prolactin, Nesfatin-1 and Ghrelin as a Marker of Epileptic Attacks Young Male Patients. Peptides, 32, 1276-1280. http://dx.doi.org/10.1016/j.peptides.2011.04.021

[17] Cağlayan, F., Cakmak, M., Cağlayan, O. and Cavuşoglu, T. (2003) Plasma D-Lactate Levels in Diagnosis of Appendicitis. Journal of Investigative Surgery, 16, 233-237. http://dx.doi.org/10.1080/08941930390215033

[18] Gavela, T., Cabeza, B., Serrano, A. and Casado-Flores, J. (2012) C-Reactive Protein and Procalcitonin Are Predictors of the Severity of Acute Appendicitis in Children. Pediatric Emergency Care, 28, 416-419. http://dx.doi.org/10.1097/PEC.0b013e318252d875

[19] Groselj-Grenc, M., Repse, S., Dolenc-Strazar, Z., Hojker, S. and Derganc, M. (2007) Interleukin-6 and Lipopolysaccharide-Binding Protein in Acute Appendicitis in Children. Scandinavian Journal of Clinical and Laboratory Investigation, 67, 197-206. http://dx.doi.org/10.1080/00365510601010397

[20] Huckins, D.S., Simon, H.K., Copeland, K., Spiro, D.M., Gogain, J. and Wandell, M. (2013) A Novel Biomarker Panel to Rule out Acute Appendicitis in Pediatric Patients with Abdominal Pain. American Journal of Emergency Medicine, 3, 1368-1375. http://dx.doi.org/10.1016/j.ajem.2013.06.016

[21] Kanat, B.H., Ayten, R., Aydın, S., Girgin, M., Cetinkaya, Z., Ilhan, Y.S., Yur, M. and Catak, Z. (2014) Significance of Appetite Hormone Ghrelin and Obestatin Levels in the Assessment of the Severity of Acute Pancreatitis. Turkish Journal of Gastroenterology, 25, 309-313. http://dx.doi.org/10.5152/tjg.2014.4081

[22] Brailoiu, G.C., Dun, S.L., Brailoiu, E., Inan, S., Yang, J., Chang, J.K. and Dun, N.J. (2007) Nesfatin-1: Distribution and Interaction with a G Protein-Coupled Receptor in the Rat Brain. Endocrinology, 148, 5088-5094. 
http://dx.doi.org/10.1210/en.2007-0701

[23] Stengel, A., Goebel-Stengel, M., Jawien, J., Kobelt, P., Taché, Y. and Lambrecht, N.W. (2011) Lipopolysaccharide Increases Gastric and Circulating NUCB2/Nesfatin-1 Concentrations in Rats. Peptides, 32, 1942-1947. http://dx.doi.org/10.1016/j.peptides.2011.07.006

[24] Stengel, A., Goebel-Stengel, M., Wang, L., Shaikh, A., Lambrecht, N.W., Rivier, J. and Tache, Y. (2011) Abdominal Surgery İnhibits Circulating Acyl Ghrelin and Ghrelin-O-Acyl Transferase Levels in Rats: Role of the Somatostatin Receptor Subtype 2. American Journal of Physiology_Gastrointestinal and Liver Physiology, 301, G239-G248. http://dx.doi.org/10.1152/ajpgi.00018.2011 